\title{
Tamarin Colon Adenocarcinoma
}

National Cancer Institute

\section{Source}

National Cancer Institute. Tamarin Colon Adenocarcinoma. NCI Thesaurus. Code C134964.

Adenocarcinoma arising in the colon of a tamarin. 